mailed to rheumatologists (adult practice) $(\mathrm{n}=995)$ who were identified through the ACR membership directory. Only physicians currently in practice were included.

Results 375 (37.7\%) questionnaires were returned between March 12, 2000 and April 25, 2000. 280 qualified for analysis. The results (A: No baseline steroid use, B: $5 \mathrm{mg} / \mathrm{d}$ prednisone) below illustrates the frequency with which rheumatologists cited each drug or drug combination as their first choice of treatment for each scenario. The most common change in medication for both cases was addition/adjustment of prednisone (A: $n=248$; $\mathrm{B}: \mathrm{n}=229, \mathrm{C} 2=4.58, \mathrm{p}=0.03)$. Of those given oral steroids for a limited amount of time, mean increase in daily dose of prednisone was A: $16.4 \pm 12.5 \mathrm{mg}$, B: $18.3 \pm 12.5 \mathrm{mg}$. Duration of increased dose was highly variable ranging from 1 day (bolus) to 3 months. Some rheumatologists chose to continue increased prednisone indefinitely, (A: $\mathrm{n}=19 ; \mathrm{B}: \mathrm{n}=14$ ). There appeared to be no consistent relationship between steroid dose and duration. For both cases there was no difference in the number of rheumatologists who increased MTX dose (A: $n=84 ; \mathrm{B}: \mathrm{n}=$ 85). The number of rheumatologists who added another DMARD to the patient's regimen were more in group A and differed significantly $(\mathrm{x} 2=3.97, \mathrm{p}=0.046$ ) from group B. Similarly, rates of initiation of anti-TNF therapy was higher in group A $(\mathrm{x} 2=4.68, \mathrm{p}=0.03)$. However, rates of addition of leflunomide showed no statistically significant difference.

Conclusion Though the cases presented were accepted by rheumatologists as RA flare requiring intervention; no consistency of practice other than an increase in steroids could be identified. Further studies determining optimal therapy for increases in RA activity are necessary.

\section{FRI0008 DIFFICULT TO TREAT RHEUMATOIDS RESPOND TO LEFLUNOMIDE}

FM Erkeller-Yuksel, JA Griffin. Rheumatology, Barnet and Chase Farm Hospitals NHS Trust, London, UK

\subsection{6/annrheumdis-2001.1137}

Background Leflunomide (LEF) is a new disease modifying agent for rheumatoid arthritis (RA). It was successful in treating relatively recent onset rheumatoid patients with a good safety record. ${ }^{1}$

Objectives Our aim was to assess the safety and efficacy of LEF in difficult to treat and chronic rheumatoid patients in a district general hospital.

Methods Patients with difficult to treat RA ( $\mathrm{n}=39$ ) were commenced on LEF. Patients were followed every 4 for 6 months for side effect profiles and efficacy recordings. We documented all side effects of LEF, routine blood test results and blood pressure measurements. Pre-treatment and 6th month erythrocyte sedimentation rate (ESR) and haemoglobin $(\mathrm{Hb})$ levels, patients' and doctors' global disease activity Visual Analogue Scores (VAS) were Statistically compared.

Results Mean age of patients was $48+14$ and duration of disease was $13+10$ years. Combination of methotrexate with LEF was the choice of treatment for 12 patients. Until now 25 patients completed 6 months evaluation and 9 out of 39 patients discontinued LEF due to side effects or infections. 6 further patients discontinued LEF after 6 months. Alopecia, diarrhoea and rash were the most common side effects $(28 \%, 26 \%$ and $23 \%$ respectively). The most serious adverse effect was sensory neuropathy of the lower limbs in a young male patient. This occurred within
10 months of LEF treatment and showed some improvement 2 months after discontinuation. Another elderly male was admitted to hospital twice with severe abdominal pains and diverticulitis.

Patients felt better on LEF treatment and showed significant improvement in reduction of ESR $(\mathrm{p}<0.02)$, increase of $\mathrm{Hb}(\mathrm{p}$ $<0.02)$ and a reduction of VAS of patients' $(0.0001)$ and doctors' ( $\mathrm{p}<0.0001$ ) global disease activity over a 6 month period (Wilcox Rank Sum Test). Due to clinical improvement we were able to stop or reduce dosage of other concominant treatment. Conclusion In conclusion LEF was reasonably well tolerated by difficult to treat rheumatoids even though side effects were more frequent than published studies (1). The clinical response was good considering the long disease duration and history of poor response to treatment in our group.

\section{REFERENCE}

1 Emery P, Breedvekd FC, Lemmek EM, Kaltwasser JP, Dawes PT, et al. A comparison of the efficacy and safety of leflunomide and methotrexate for the treatment of rheumatoid arthritis. Rheumatology 2000;39:655-65

\section{FRI0009 INFLIXIMAB IN THE TREATMENT OF RHEUMATOID ARTHRITIS (RA): REFERRAL PATTERNS, RESPONSE TO TREATMENT, AND ADVERSE EVENTS IN A REAL WORLD SETTING}

Y Yazici, D Erkan, I Kulman, L Leff, J Markenson, MJ Harrison. Rheumatology, Hospital for Special Surgery, New York, USA

\subsection{6/annrheumdis-2001.1138}

Background Infliximab is a chimeric, monoclonal antibody against tumour necrosis factor (TNF) alpha, recently developed to complement methotrexate therapy in RA patients with a less than optimal response to treatment. To date it has only been studied in clinical trials.

Objectives To describe referral patterns, patient response to treatment, and adverse events in a cohort of RA patients treated with infliximab in a real world setting.

Methods Patients who had received their first dose of infliximab between $1 / 00$ and $6 / 00$ were included to ensure the possibility of at least 6 months of drug exposure. We evaluated patients at baseline, and followed them prospectively every 2 months for a total of 6 months. Patients completed several questionnaires regarding RA history, treatment, and disease activity (HAQ, pain, fatigue, tender and swollen joint counts) at each infusion. All subjects were contacted between infusions for reporting of side effects.

Results 36 patients were treated with infliximab between January 1 - June 30, 2000 (female $\mathrm{n}=33$, mean age $=63 \pm 12.9 \mathrm{y}$, disease duration $=15.3 \pm 10.2 \mathrm{y}$ ). They had previously failed an average of 3 DMARDs. 24 patients were on oral steroids at baseline. 16 patients received infliximab without concurrent MTX. 12 of 36 patients discontinued treatment before 6 months. Discontinuation reasons were inadequate response $(\mathrm{n}=$ $5)$, repeated episodes of dyspnea $(n=3)$, asthma $(n=1)$, chest tightness $(\mathrm{n}=1)$, phlebitis $(\mathrm{n}=1)$, and patient noncompliance $(n=1)$. Table 1 shows individual measures of disease activity in 24 patients who completed 6 months of infliximab. We detected a significant improvement in HAQ scores, and trends toward improvement in pain scores and tender joint counts. HAQ scores improved in $19 / 24$, stiffness in $14 / 24$, pain in $13 / 24$, fatigue in $13 / 24$, tender joint counts in $13 / 24$ and swollen joints in $15 / 24$ of patients. Since initiating infliximab therapy, of the 36 patients receiving 198 infusions, 18 patients reported a total of 33 